\title{
Marketing digital e de relacionamento: Avaliação de um sistema de informação digital usando uma integração da TAM, TTF e modelos KMV
}

Relationship and digital marketing: Evaluation of a digital information system using an integration of TAM, TTF and KMVmodels

Plínio Rafael Reis Monteiro ${ }^{1}$, Raquel Vaz de Mello Strambi Zeringota ${ }^{1}$

Universidade Federal de Minas Gerais.

\section{RESUMO}

A ascendente popularidade da internet prolifera formas de entretenimento, comunicação e trabalho, tais como os portais, que visam difundir informações e fomentar relacionamentos entre stakeholders. Este estudo buscou verificar como a interação tecnológica do portal de uma IES afeta atitudes (satisfação, confiança e comprometimento) e intenções comportamentais (utilização do portal, lealdade e comunicação positiva) dos discentes. A partir de um survey concebido pelos modelos TAM, TTF e KMV o estudo evidencia que as características e a adequação da tecnologia influenciam na utilidade percebida no portal e nas atitudes moderadoras de intenções comportamentais para a instituição estudada.

PALAVRAS-ChAVE: Portal; Digital, Avaliação de sistema de recuperação de informação

\begin{abstract}
The increasing popularity of internet promotes new forms of entertainment, communication and work, such as portals, which aim to disseminate information and foster relationships among stakeholders. This study sought to demonstrate how technological interaction portal of an Educational Institution affects attitudes (satisfaction, trust and commitment) and behavioral intentions (usage level, loyalty and positive communication) of students. From a survey designed by TAM, TTF and KMV models the study showed that the characteristics and suitability of technology on perceived influence in moderating the portal and attitudes of behavioral intentions to study hospital use.
\end{abstract}

KEYWORDS: Portal; Digital, Evaluation of information retrieval system

\section{Correspondência}

1Plínio Rafael Reis Monteiro

Universidade Federal de Minas Gerais.

Belo Horizonte, MG.

Email: preisufmg@gmail.com

ORCID: http://orcid.org/0000-0002-5626-2945

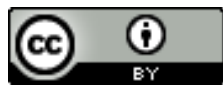

JITA: FB. Marketing 


\section{INTRODUÇÃ̃O}

Vivemos uma era em que o conhecimento e a informação se tornam recursos intangíveis centrais (AMARAL, 2008), onde a capacidade de gerenciar e transformar dados em vantagens competitivas para o fomento de relacionamentos entre organizações e seu público (KIM H; DUNNIGAN, 2014) se tornou imprescindível. No Brasil contemporâneo, esse cenário se torna mais proeminente a partir do avanço da internet, que alcançou 77,8 milhões de pessoas no segundo trimestre de 2011 (MEIO E MENSAGEM, 2011), e sua influência em diversas searas da vida em sociedade.

A expansão da internet, em especial pela proliferação das redes sociais e aplicativos, tem revolucionado o relacionamento das organizações com diversos stakeholders, com destaque para a relação com o mercado. Cada vez mais o cliente, intrincado nas interseções entre o ambiente off e online, passa a se relacionar com marcas e organizações mediado por estas tecnologias (TERRA, 2010), revelando novos desafios de marketing para as organizações (SANTOS; FRANCO; TERRA, 2009).

O relacionamento entre organizações e consumidores não é um tema recente na literatura, apesar de sua crescente popularidade (PARVATIYAR; SHELT, 1999). O termo marketing de relacionamento exemplifica esta importância, representando práticas, processos e filosofias gerenciais que visam criar valor compartilhado entre organizações e consumidores individuais com vistas a fomentar relacionamentos duradouros e profícuos mutuamente (GUMMESSON, 1999; HOLLENSEN, 2010). Deste modo, em uma sociedade digital e interconectada, a prática do marketing de relacionamento prescinde progressivamente do uso de ferramentas digitais com vistas a promover o relacionamento com seu público (LEEFLANG et al., 2014).

Uma das formas de se estabelecer um contato on-line com os clientes é criar e manter um portal. Considerado um site que se diferencia dos demais, devido a seu tamanho e volume de conteúdo disponível, o portal inclui ferramentas digitais, links para redes sociais, como Twitter, Facebook, Linkedin, capazes de interagir e se relacionar com o seu público, bem como outras ferramentas administrativas/gerenciais a disposição de diferentes stakeholders. Também são uma forma de reter e compartilhar o conhecimento e, principalmente, a superação de alguns desafios para as empresas, como, por exemplo, manter um relacionamento duradouro com o cliente por meio de uma plataforma digital e obter retorno sobre investimentos realizados.

Este desafio se aplica indubitavelmente as IES devido a inúmeros fatores, tais como a mudança do perfil dos alunos, a expansão da demanda, o impacto de novas tecnologias no contexto educacional, a emergência de novas práticas de ensino e o aumento da concorrência (TOLENTINO, R. DE S. DA S. et al., 2013). Deste modo, a utilização dos portais como ferramenta de marketing de relacionamento trata de um fenômeno recente e ao mesmo tempo desafiador. Neste aspecto, foi proposta a seguinte pergunta-chave para esta pesquisa: qual o papel dos portais na manutenção do relacionamento com os alunos de uma IES?

\begin{tabular}{|c|c|c|c|c|c|}
\hline (C) RDBCI: Rev. Digit. Bibliotecon. Cienc. Inf. & Campinas, SP & v. 15 & n. & p. $189-210$ & jan./abr. 2017 \\
\hline
\end{tabular}


Deste modo, expõem-se como objetivo deste trabalho verificar como a interação tecnológica do portal de uma IES afeta atitudes (satisfação, confiança e comprometimento) e intenções comportamentais (utilização do portal, lealdade e comunicação positiva) dos discentes. Adotou-se como abordagem teórica o Modelo de Aceitação de Tecnologia (Technology Acceptance Model - TAM), formulado por Davis (1989), o Modelo de Adequação da Tecnologia (Task Technology Fit - TTF), baseado na teoria de Goodhue e Thompson (1995), e o modelo dos Moderadores Chave do Relacionamento (Key Moderator Variables - KMV), adaptado de Morgan e Hunt (1994).

Como as organizações investem vultuosos recursos na manutenção de portais, tendo, dentre suas premissas, a de que tal ferramenta poderá fomentar um relacionamento duradouro com seus stakeholders, a pesquisa poderá revelar a contribuição destes investimentos na formação de atitudes (satisfação, confiança e comprometimento) e intenções comportamentais dos usuários (de uso do portal). Ademais, o modelo propõe uma expansão do conhecimento ao relacionar variáveis típicas de modelos de intenção de uso de tecnologia (TAM e TTF) com construtos típicos do marketing de relacionamento.

\section{REFERENCIAL TEÓRICO}

\subsection{Marketing de relacionamento e marketing digital}

A partir da década de 1980, o marketing de relacionamento começou a ganhar atenção dos pesquisadores com o aumento da concorrência e a percepção de que manter clientes é mais barato, mais simples e mais lucrativo do que conquistar novos (NAKAGAWA; GOUVÊA, 2006).

Para Parvatiyar e Shelth (2001), marketing de relacionamento é uma filosofia dos negócios que prima por estabelecimento, manutenção e melhoria das relações com clientes e parceiros de forma rentável para ambas as partes. O foco do marketing de relacionamento são as relações de longo prazo entre vendedor-comprador e demais stakeholders que mantêm laços diretos ou indiretos com a empresa e que se sustentam na colaboração e na cooperação entre as partes.

Deve-se destacar que o relacionamento entre compradores e vendedores é o terreno para o marketing em sua abordagem relacional ou transacional. De acordo com o pensamento tradicional do marketing, as trocas comerciais ocorrem de forma impessoal, por meio de promoção e distribuição de massa. Empresas operando nessa lógica oferecem produtos e serviços por meio de intermediários, enquanto o cliente oferece dinheiro. Nessa perspectiva "restrita" os consumidores são considerados como números, apenas dados estatísticos. Paralelamente, o objetivo do marketing de relacionamento são trocas de longo prazo que agregam valor tanto para o comprador como para o vendedor (GUMMESSON, 2002). Neste cenário, a busca pela conservação de trocas relacionais ganha ímpeto na profusão da internet e sua importância no cotidiano social e empresarial, aproximando o campo do marketing de 
relacionamento ao denominado marketing digital.

O marketing digital pode ser definido como a utilização das ferramentas disponíveis na internet como formas de se suprir as necessidades do consumidor e atingir o objetivo da empresa. Para isso, é preciso envolver publicidade, propaganda e todos os conceitos já conhecidos nas teorias mercadológicas (TORRES, 2009).

Com o crescimento de usuários conectados à internet, é necessário incluir o marketing digital no planejamento estratégico das empresas. A importância de se estudar sites de empresas é o estabelecimento, a manutenção ou a consolidação dos processos de comunicação entre a organização e o cliente, que poderão desencadear uma transação comercial. (CARVALHO; CARVALHO; CARVALHO, 2007).

Segundo Agustini e Minciotti (2003), empresas devem criar novas relações com o consumidor e identificar por que ele prefere comprar pela internet ao invés de ir até a loja. A interatividade, oferecida pela Web 2.0, a conectividade, possível graças a sistemas on-line, e a criatividade auxiliam a obtenção de informações que ajudam a gerenciar o relacionamento com o consumidor. Estabelecer um diálogo contínuo resulta em um relacionamento duradouro, no conhecimento do perfil psicográfico, do estilo de vida e do comportamento de compra do cliente.

Surge um novo desafio para as organizações: trabalhar com eficácia no mundo virtual, pois consumidores e novos profissionais que ingressarão no mercado de trabalho já nascem com um DNA digital e familiarizados com essas novas ferramentas (TERRA, 2010). Na internet, os concorrentes estão a apenas um clique de distância, o que faz que esse ambiente seja ainda mais competitivo e vulnerável. Os clientes avaliam a sua experiência on-line por meio de comentários e retornam aos sites caso sua experiência tenha sido positiva (FARIAS, 2006).

Atualmente, a vantagem competitiva de uma empresa está muito vinculada ao seu capital de relacionamento traduzido por reputação, confiança e construção das redes. Esses conceitos devem ser aplicados no relacionamento entre clientes, fornecedores, parceiros e funcionários. Um estudo desenvolvido por Hernandez (2008) percebeu que a falta de confiança em relação a transações realizadas por comércio eletrônico era uma das primeiras barreiras ao crescimento dessa área. A Política de Privacidade dos sites é uma questão de preocupação para as empresas e os clientes. Pela internet, é possível rastrear informações sobre o comportamento e o perfil do internauta; relacionamentos construídos ao longo de muitos anos podem ficar comprometidos, caso o consumidor tenha suas informações pessoais expostas. (CARVALHO; CARVALHO; CARVALHO, 2007).

De acordo com Alves, Lamounier e Jabur (1999), uma pane nos sistemas on-line das empresas causa significativos prejuízos em sua imagem, arranha sua credibilidade no mercado e pode ser passível de uma ação judicial por parte dos usuários. Balarine (2002)

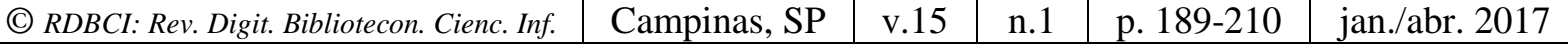


defende que optar por grandes investimentos em Tecnologia da Informação, a fim de desenvolver projetos por impulsos ou modismos, é arriscado.

Pode-se notar que a relação entre clientes e organizações perpassa progressivamente as interações ocorridas no ambiente virtual, de modo que os temas marketing de relacionamento e marketing digital estarão cada dia mais associados, se tornando imponderável conceber estratégias de geração de valor compartilhado sem a mediação da internet, por meio de redes sociais, sites, aplicativos e portais (HOLLENSEN, 2010; LEEFLANG et al., 2014)

\subsection{Portais}

Os portais são uma forma de reter e compartilhar o conhecimento e, principalmente, de superar alguns desafios para as empresas, como, por exemplo, manter um relacionamento duradouro com o cliente por meio de uma plataforma digital e obter retorno sobre investimentos realizados para o desenvolvimento e manutenção de um portal. Eles são caracterizados pelo grande volume de informação que armazenam, diferenciando-se de um simples site da internet. Também possibilitam criação e reutilização do conhecimento, sistematização dos processos de geração de conteúdos e são fontes de conhecimentos. Para 30 que alcancem esses objetivos, os portais devem oferecer motivos para que os usuários os visitem diariamente (TERRA; GORDON, 2002)

Os portais possuem um potencial para que as empresas embarquem em transformações significativas de seus modelos de negócios, sendo uma base para o desenvolvimento de relacionamentos além dos limites da empresa. Decorrente dessa abordagem, ocorrem ciclos de inovação mais rápidos, melhores ambientes, melhor atendimento ao cliente, estabelecimento de parcerias, receitas maiores e custos reduzidos. Podem-se acrescentar como benefícios dos portais: fluxo de informação e conhecimento, maior integração, abordagem ampla, coordenada e com base em projetos, conexões entre as pessoas e entre pessoas e informação (TERRA; GORDON, 2002).

As empresas devem-se preocupar com boa usabilidade, permitindo ao usuário atingir o seu objetivo, chegando ao resultado pretendido com eficiência, ou seja, com qualidade e competência, sem perdas ou desperdícios de tempo e sem erros de percurso. (MARTINEZ; FERREIRA; GALINDO, 2011). Apesar do grande avanço que as tecnologias da informação obtiveram na última década, a questão da usabilidade não conseguiu acompanhá-la, pois essa é uma questão de comportamento humano, e as pessoas não mudam radicalmente em apenas uma década (NIELSEN; LORANGER, 2007).

Também a acessibilidade deve ser considerada quando se trabalha com a rede mundial de informação, a internet. Contemplar controle sobre tamanho de fonte, para usuários com dificuldade de visão, como idosos, míopes ou, em caso de cegos, navegação com vocalizadores, é um dos princípios da acessibilidade.

Para Nakagawa e Gouvêa (2006), a experiência de compra de um consumidor on-line

\begin{tabular}{|c|c|c|c|c|c|}
\hline (C) RDBCI: Rev. Digit. Bibliotecon. Cienc. Inf. & Campinas, SP & v.15 & n.1 & p. $189-210$ & jan./abr. 2017 \\
\hline
\end{tabular}


compreende três elementos principais: qualidade da interface, qualidade do encontro e qualidade da execução, interpretadas como processo, experiência e resultados, itens em que os autores sugerem excelência por parte das organizações.

\subsection{Modelos de Uso de Tecnologia da Informação}

\subsubsection{Modelo TAM}

A falta de aceitação dos usuários tem sido um impedimento para o sucesso dos sistemas de informação. Para explicar por que usuários aceitam ou rejeitam um sistema de informação e, quando aceitam, quais características do sistema favorecem essa aceitação, Davis (1989) propôs um modelo de aceitação tecnológica (TAM) baseado nos princípios da Teoria da Ação Racionalizada, criada por Fishbein e Ajzen (1975), que defende que crenças levam a atitudes que desencadeiam intenções que culminam em comportamentos. O objetivo do TAM é trazer uma referência para o impacto de fatores externos nas crenças internas, atitudes e intenções. (Fishbein e Ajzen, 1975, apud Vasconcellos, 2008)

A proposta do TAM é mostrada na FIG.1. Atitudes que levam ao uso de um sistema provêm de duas crenças: a facilidade de uso e a utilidade percebida. O design do SI influencia diretamente a facilidade de uso e a utilidade percebida (DAVIS, 1989).

De acordo com Davis (1989), a facilidade de uso pode ser entendida para o usuário como quanto de esforço um sistema demandará. A utilidade percebida é mensurada como quanto o uso de um sistema melhorará o desempenho do indivíduo na realização de uma_tarefa.

Segundo uma pesquisa realizada por Davis (1993), a variável utilidade percebida é mais significativa e forte do que a facilidade percebida. A Utilidade Percebida é influenciada por diversos fatores externos, independente da Facilidade Percebida. Por exemplo, dois sistemas igualmente fáceis de operar, se um apresentar melhores resultados, então será apresentado como tendo uma utilidade percebida melhor que o outro. Um sistema que apresenta melhores resultados e for mais difícil de operar será percebido como mais útil do que aquele que não apresenta bons resultados (SALEH, 2004).

O TAM possui um questionário que será utilizado nesta pesquisa cujas perguntas foram criadas a partir dos indicadores presentes neste modelo. São definidos como indicadores do TAM: ambiguidade e interdependências nas tarefas, utilidade percebida, intenção de uso do site e característica da tecnologia. 
Figura 1. Modelo de Aceitação Tecnológica (TAM)

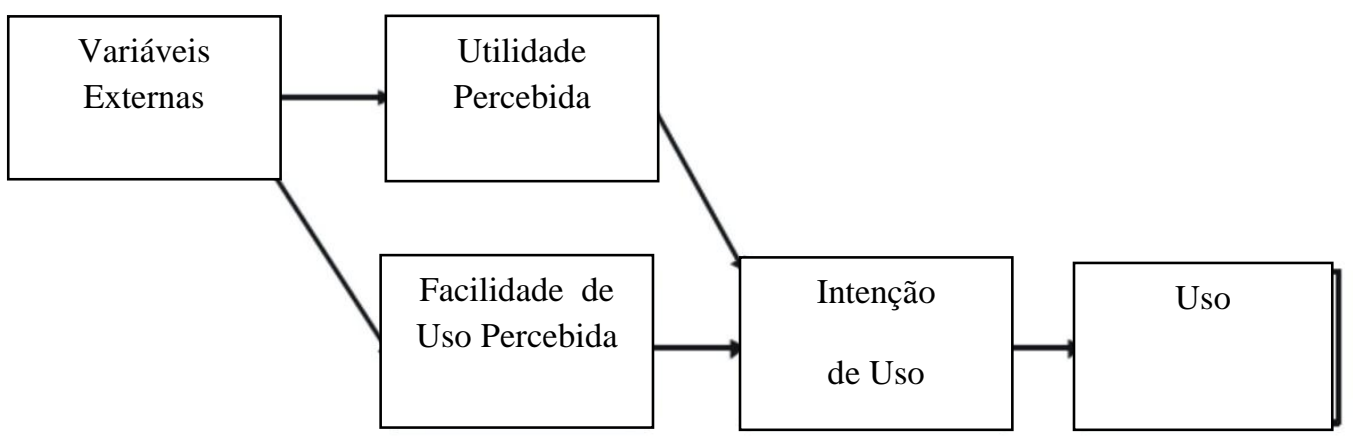

Fonte: DAVIS, 1989, p8

\subsubsection{Modelo Task Technology Fit (TTF)}

O modelo Task Technology Fit propõe que a adequação da tecnologia à tarefa impacta na performance. $\mathrm{O}$ foco na adequação possui evidências em pesquisas que demonstraram, após uma série de experimentos em laboratórios, que o impacto no desempenho depende da adequação da tecnologia à tarefa. Sistemas de informação somente têm impactos positivos na performance se há uma adequação entre suas funcionalidades e a necessidade requerida pela tarefa (GOODHUE; THOMPSON, 1995).

Sugere-se, também, uma ligação entre adequação do sistema e utilização. Em um nível organizacional, elas são relacionadas: no nível individual, a adequação ao trabalho é um forte constructo que prediz o uso da tecnologia em uma estação de trabalho (GOODHUE; THOMPSON, 1995).

Esse modelo quando adotado separadamente possui algumas limitações. Para alguns usuários, utilizações de alguns sistemas indicam mais a designação do trabalho do que sua qualidade, utilidade ou atitudes favoráveis à sua utilização (GOODHUE; THOMPSON, 1995).

O modelo TTF contém os seguintes constructos: qualidade, acesso, autorização, compatibilidade, eficiência no tempo de produção, confiabilidade no sistema, facilidade de uso, treinamento, consultoria e relacionamento com usuários.

O indicador qualidade pode ser traduzido como o quão atualizadas, corretas e confiáveis são as informações do portal, com níveis de detalhes apropriados para que o usuário o utilize. Acesso diz respeito a facilidade de encontrar dados e entender a linguagem usada. O indicador autorização pode ser entendido como o quanto informações importantes e úteis estão disponíveis. A compatibilidade retrata o quanto as informações se apresentam coerentes e alinhadas com outras fontes de conteúdo.

Já o constructo eficiência no tempo de produção indica o quanto as informações importantes são publicadas rápidas no site. A confiabilidade do site implica no quanto o sistema está disponível no ar, não apresentando erros frequentes e lentidão na sua navegação.

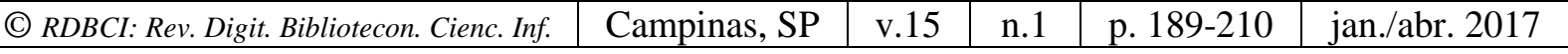


A facilidade de uso indica o quanto o portal apresenta uma navegação intuitiva, sendo a lógica o mínimo de esforço para se atingir o objetivo proposto. A consultora é um indicador que visa sinalizar o quanto o aluno acredita que obterá o apoio do departamento de informática caso precise. E, por último, indicar o TTF possui o relacionamento com usuário que objetiva demonstrar o quanto o site atende as demandas dos alunos, por meio de respostas rápidas e certas.

Dishaw e Strong (1999, apud KLOPPING; MCKINNEY, 2004) demonstraram a eficácia de se combinar os modelos TAM e TTF na adoção de tecnologias. Separadamente, o TTF é mais eficaz em explicar tarefas de trabalho, mas, combinados, eles se tornam um modelo superior capaz de explicar melhor o comportamento do usuário na adoção da tecnologia. O TTF afeta a facilidade de uso, mas surpreendentemente não afeta a utilidade de uso. Nesta pesquisa, utilizou-se o modelo TAM combinado com o modelo TTF conforme mostrado na Figura 2.

Figura 2. Combinação do modelo TAM + TTF -

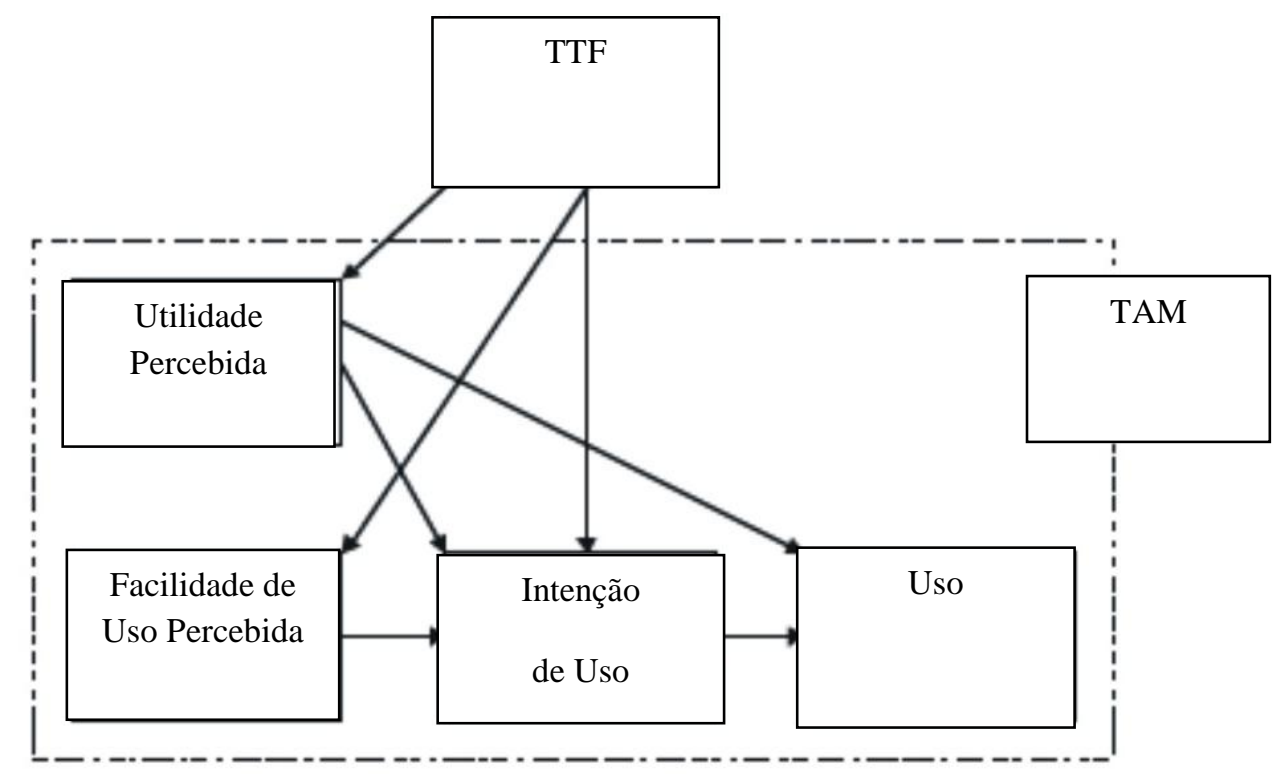

Fonte: Klopping e Mckinney (2004).

\subsubsection{Modelo de Moderadores Chave do Relacionamento}

A década de 1980 foi testemunha da maior mudança no marketing de relacionamento tanto na teoria quanto na prática. Com a crescente concepção das limitações das práticas predatórias de competição, muitas empresas estão aprendendo que devem atuar de forma colaborativa com fornecedores, distribuidores e clientes com vistas a garantir retornos sustentáveis aos membros de uma cadeia de suprimentos (MORGAN; HUNT, 1994). 
Essa filosofia emergente pressupõe que haja um fundamento de interesses e acordos mútuos, em que o mercado não é uma selva, mas sim um sistema complexo e interdependente e, por isso, fundamentalmente cooperativo (MORGAN; HUNT, 1994).

Manter e aprofundar os vínculos com os clientes são tarefas que exprimem de forma o principio do marketing de relacionamento, onde a essência é a troca profícua e duradoura entre os participantes. Dwyer, Schurr e Oh (1987, apud MORGAN; HUNT, 1994) propõem que o marketing de relacionamento se refere a todas as atividades de marketing direcionadas a estabelecer, desenvolver e manter trocas relacionais de sucesso (MORGAN; HUNT, 1994).

$\mathrm{Na}$ área de comunicação, um construto-chave é a credibilidade, originalmente definida como a confiança no locutor pelo ouvinte. A eficácia nos serviços depende do gerenciamento da confiança, porque o cliente compra um serviço antes de experimentá-lo. Nas empresas, a exigência pela confiança é a base da lealdade. Morgan e Hunt (1994) afirmam que a parceria com qualquer um implica uma parcela de risco e recompensa, e jogos que visam ao ganho de ambas as partes exigem confiança mútua.

A confiança influencia o comprometimento com os relacionamentos, porque relacionamentos baseados na confiança são tão valiosos que as partes querem se comprometer com eles (MORGAN; HUNT, 1994).

Após duas décadas de estudos e pesquisas sobre comprometimento no campo organizacional, Morgan e Hunt (1994) identificaram os mais importantes precursores de relacionamentos baseados na confiança e no comprometimento: custos de término de relacionamentos e benefícios nos relacionamentos influenciam diretamente $\mathrm{o}$ comprometimento, valores influenciam compromisso e confiança dos relacionamentos e comunicação e comportamento oportunista influenciam a confiança que indiretamente influencia o comprometimento (MORGAN; HUNT, 1994). Este estudo considerou o modelo de Hunt e Morgan como base da pesquisa de marketing de relacionamento, empregando variáveis mediadoras (satisfação, comprometimento e confiança) e suas consequências (propensão a lealdade e boca a boca positivo), como consequências das características da tecnologia e suas aplicações, propostas nos modelos TAM e TTF.

\subsection{Construção do modelo de pesquisa}

Conforme demonstrado previamente, a base deste modelo é a conjugação dos modelos de adoção de tecnologias (TAM +TTF) e de relacionamento (KMV), sob a premissa de que a tecnologia digital, representada neste caso pelo portal, emerge como um importante alicerce na construção das bases relacionais entre a organização e seu público alvo.

Conforme citam Klopping e Mckinney (2004), tanto a adequação da tecnologia, expressa no modelo TTF, quanto suas características são relevantes para que sua utilização. A junção destes modelos de pesquisa da origem as hipóteses concebidas originalmente por Goodhue e Thompson (1995) e Davis (1989) em uma estrutura proposta por Tolentino et al.

\begin{tabular}{|c|c|c|c|c|c|}
\hline (C) RDBCI: Rev. Digit. Bibliotecon. Cienc. Inf. & Campinas, SP & v.15 & n.1 & p. $189-210$ & jan./abr. 2017 \\
\hline
\end{tabular}


(2007), dando origem as hipóteses H1 à H4.

Já a alegação da importância da tecnologia como antecedente e ponto central de relacionamento na ótica do marketing de relacionamento tem sido recentemente debatido na literatura (HOLLENSEN, 2010; LEEFLANG et al., 2014; OKADA; SOUZA, 2011). Tais autores destacam a importância que as ferramentas digitais exercem para fomentar relacionamentos entre as organizações e o mercado alvo (NAKAGAWA; GOUVÊA, 2006). Estas relações perpassam necessariamente por mediadores chave no contexto de marketing de relacionamento (MORGAN; HUNT, 1994). Neste sentido, a proposição de integração consiste justamente em interligar a adequação e as características da tecnologia como sorvedouros de satisfação, confiança e comprometimento dos usuários/clientes. Essa assertiva sustenta as hipóteses H5 às H17 do modelo de pesquisa.

$\mathrm{Na}$ ótica do marketing de relacionamento, tipicamente as variáveis fim representam consequências comportamentais como a propensão a lealdade e a comunicação positiva (boca a boca). No modelo KMV, sugere-se que a satisfação, mas em especial, a confiança e o comprometimento, representam os mediadores das bases de cunho transacional, neste caso, inerentes a tecnologia, para os resultados esperados na gestão do relacionamento. Esta constatação, embasada em Alejandro et al. (2011) e Vieira (2011), sustenta as hipóteses H19 às H21 e H23 às H25. Finalmente, as hipóteses H22 e H18 referem-se ao impacto direto da utilidade percebida no sistema como antecedente de lealdade e boca a boca positivo, em consonância com a premissa de que a utilidade refere-se a um construto atitudinal de origem cognitiva (DAVIS, 1989), de forma diversa da satisfação que tem natureza afetiva. Assim, ambos construtos representam antecedentes comportamentais que captam aspectos distintos, mas complementares, do efeito que a tecnologia para fomentar o relacionamento entre os alunos e a organização estudada. As hipóteses citadas são apresentadas na Figura 3, representadas pelas setas no modelo estrutural testado, e citadas detalhadamente na tabela 2.

\section{METODOLOGIA}

Este estudo foi do tipo descritivo com corte transversal, quantitativo, pela aplicação de um levantamento (survey) on-line com alunos de uma universidade privada de uma capital brasileira. A unidade de análise do estudo foi o novo portal na internet da instituição, bem como seus sistemas de informação acadêmica, tendo sido coletados 158 questionários com alunos de graduação e pós-graduação, por meio de uma amostragem de conveniência. A amostra foi considerada satisfatória de acordo com os critérios propostos por Chin (2000), que indicaria uma amostra mínima de 120 casos a serem pesquisados.

O questionário empregou escalas do tipo Likert de cinco pontos (1 a 5), amplitude suficiente para captar a variabilidade dos construtos e capaz de gerar entendimentos mais nítidos das opções de respostas, dado que todos os pontos foram rotulados (escala verbal). As escalas foram adaptadas de autores prévios, buscando modificar o fraseado para se adaptar a as funcionalidades do portal e ao perfil da IES. As escalas compreenderam elementos

\begin{tabular}{|c|c|c|c|c|c|}
\hline (C) RDBCI: Rev. Digit. Bibliotecon. Cienc. Inf. & Campinas, SP & v.15 & n.1 & p. $189-210$ & jan./abr. 2017 \\
\hline
\end{tabular}


relacionais (Comprometimento, Satisfação, Confiança, Lealdade, Comunicação Boca a boca Positiva) e de interação/adoção de modelos de tecnologia (Características da tarefa, Características da Tecnologia, Facilidade de Uso, Adequação da Tecnologia, Utilidade), quais sejam; Modelo de Aceitação da Tecnologia (TAM) (LACERDA; MENDONÇA, 2009), Modelo de Adequação da Tecnologia (KLOPPING; MCKINNEY, 2004; GOODHUE; THOMPSON, 1995), Modelo dos Moderadores Chave do Relacionamento (KMV) (VIEIRA; MONTEIRO; VEIGA, 2011; MORGAN; HUNT, 1994).

Para análise de dados empregou-se o SPSS® e o SmartPLS para análises de pressupostos, adequação, qualidade das medições e para teste do modelo por intermédio da modelagem de equações estruturais. A modelagem de equações estruturais foi empregada, parecia ser adequada para responder a questões sobre múltiplos relacionamentos entre variáveis e de qualidade de mensuração, enquanto a escolha da abordagem PLS se deve a complexidade do modelo, restrições quanto ao tamanho da amostra e desvios esperados da normalidade (HAIR et al., 2014).

\section{ANÁLISE DE DADOS}

A análise se iniciou pela depuração e avaliação da adequação dos dados, empregando a análise descritiva de dados ausentes (missing values), outliers (uni e multivariados), normalidade de dados e linearidade (TABACHNICK; FIDEL, 2001), onde os problemas potenciais foram considerados aceitáveis após o tratamento sugerido na literatura (HAIR et al., 2010).

Após a análise da dimensionalidade por intermédio da Análise Fatorial Exploratória (AFE) analisou-se a confiabilidade dos construtos. Para tanto, empregou-se a Confiabilidade Composta (CC) e o Alpha de Cronbach (AC) usando como ponte de corte o valor de 0,700 (HAIR et al., 2010), alcançado por todas as medidas. A confiabilidade dos indicadores foi balizada pela verificação que todas a cargas fatoriais foram superiores a 0,500 (HULLAND, 1999). Para analisar a qualidade da mensuração e confiabilidade média dos indicadores, empregou-se a Variância Média Extraída (AVE), empregando como ponto de corte o valor de 0,500 (FORNELL; LARCKER, 1981).

Na etapa confirmatória o modelo estrutural foi concebido no software SmartPLS2.0 visando testar tanto a validade de construto das medidas (NETEMEYER; BEARDEN; SHARMA, 2003). A validade convergente foi suportada pela constatação de que todas as cargas fatoriais no teste de modelo de mensuração na etapa confirmatória foram significativas $(\mathrm{p}<0,05)$. Já a validade discriminante analisada ao comparar a variância média partilhada pelos indicadores (AVE) com a partilhada pelos construtos (quadrado das correlações). Neste caso, a exceção do par "04. Adequação à tecnologia" e "06. Satisfação", todos os demais apresentaram validade discriminante. Não obstante, segundo o critério do intervalo de confiança párea as correlações corrigidas pelos erros de mensuração (correlação desatenuada), método sugerido por Netemeyer, Bearden e Sharma (2003) verificou-se que o

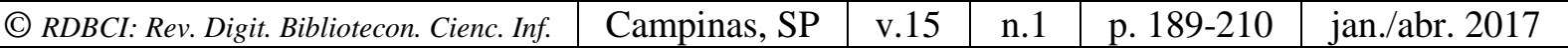


intervalo de $99 \%$ de confiança da correlação corrigida entre estes construtos não se aproxima do valor unitário (1), ou seja, a correlação entre eles é inferior a 1. Dessa forma, com base nos métodos propostos, é possível atestar a validade discriminante e convergente das medidas, conforme resume a Tabela 1 .

Tabela 1. Resumo das medidas de confiabilidade e validade das medidas

\begin{tabular}{|c|c|c|c|c|c|c|c|c|c|c|}
\hline Construtos & & & & & & & & & & $\mathbf{0}$ \\
\hline 01. Carac. da Tarefa & 1,83 & & & & & & & & & \\
\hline 02. Carac. da Tecnologia & 1,04 & 1,62 & & & & & & & & \\
\hline 03. Facilidade de uso & 1,31 & 1,16 & 1,69 & & & & & & & \\
\hline 04. Adequação à tecnologia & ।,14 & 1,45 & , 15 & ',47 & & & & & & \\
\hline 05. Utilidade & 1,03 & 1,55 & 1,08 & 1,45 & ',60 & & & & & \\
\hline 06. Satisfação & 1,05 & 1,38 & 1,06 & 1,60 & 1,38 & ।,71 & & & & \\
\hline 07. Comprometimento & 1,01 & 1,25 & 1,01 & 1,23 & 1,24 & 1,33 & ।,72 & & & \\
\hline 08. Confiança & 1,08 & 1,26 & 1,05 & 1,38 & 1,30 & ।,38 & 1,55 & ',78 & & \\
\hline 09. Propensão a Lealdade & 1,00 & 1,08 & 1,00 & 1,10 & ',07 & ।,21 & 1,50 & 1,38 & ।,73 & \\
\hline 10. Comunicação positiva & 1,04 & 1,18 & 1,03 & 1,24 & 1,18 & 1,21 & 1,57 & 1,43 & 1,47 & ।,87 \\
\hline Confiabilidade Composta & 1,91 & 1,86 & 1,87 & 1,87 & 1,82 & 1,88 & 1,93 & 1,92 & 1,89 & 1,95 \\
\hline Alpha de Cronbach & 1,80 & 1,79 & 1,81 & 1,82 & 1,67 & 1,79 & 1,9 & 1,86 & 1,81 & 1,93 \\
\hline Variância Média Extraída & 1,83 & 1,62 & 1,69 & ।,47 & 1,6 & ।,71 & ।,72 & ।,78 & ।,73 & ।,87 \\
\hline
\end{tabular}

Fonte: Dados da pesquisa.

Após esta análise, procedeu-se a avaliação das relações e hipóteses do modelo pela avaliação do Modelo dos Caminhos Internos (Inner Path Model) (HENSELER; RINGLE; SINKOVICS, 2009) que trata de uma forma de analisar a validade nomológica (ANDERSON; GERBING, 1988). Os erros padrão robustos foram estimados pelo procedimento bootstrapping e usados para calcular testes $\mathrm{T}$ unicaudais com valores críticos de 1,655 para 5\% e 2,355 para 1\%. Usando estes parâmetros, são construídas as considerações e resultados do estudo pela apresentação das hipóteses testadas pelo modelo e representadas na Figura 3, sendo que as em negrito foram confirmadas, conforme já relatado. 
Figura 3. Modelo Estrutural testado.

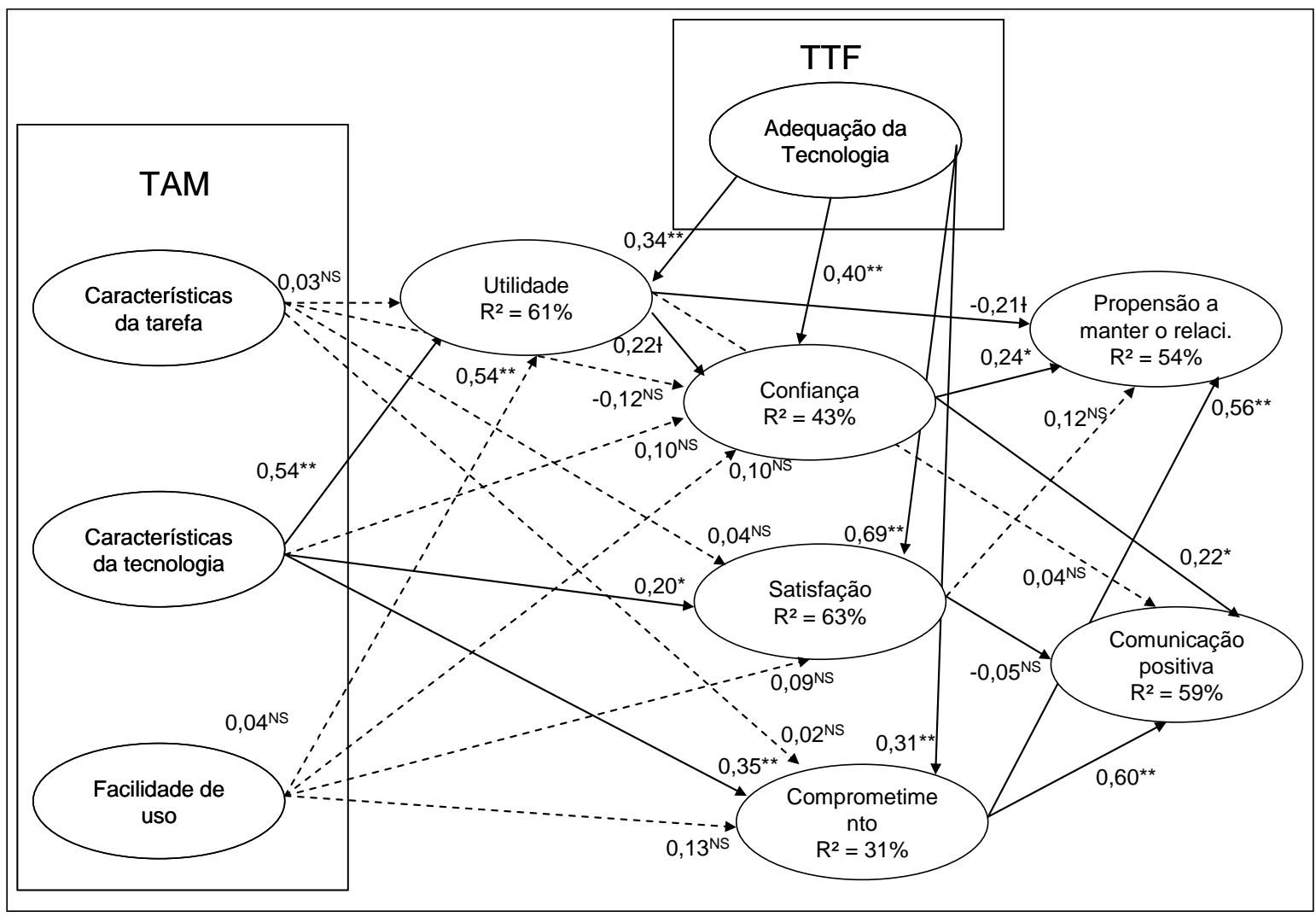

Fonte: Dados da Pesquisa

Além de representar o modelo graficamente, apresenta-se detalhadamente as relações e hipóteses testadas na hipótese 2.

Tabela 2. Testes de hipóteses do modelo

\begin{tabular}{|c|c|c|c|c|c|}
\hline Construto exógeno & $\begin{array}{l}\text { Construto } \\
\text { endógeno }\end{array}$ & Peso & Erro & Valor $\mathbf{T}$ & Teste Hipótese \\
\hline 01. Características da Tarefa & \multirow{4}{*}{ 05. Utilidade } & 0,03 & 0,08 & 0,40 & H1 - Rejeitada \\
\hline 02. Características da Tecnologia & & 0,54 & 0,10 & 5,43 & H2 - Suportada \\
\hline 03. Facilidade de uso & & 0,04 & 0,09 & 0,49 & H3 - Rejeitada \\
\hline 04. Adequação à tecnologia & & 0,34 & 0,10 & 3,38 & H4 - Suportada \\
\hline 01. Características da Tarefa & \multirow{4}{*}{ 06. Satisfação } & 0,04 & 0,09 & 0,40 & H5 - Rejeitada \\
\hline 02. Características da Tecnologia & & 0,20 & 0,12 & 1,66 & H6 - Suportada \\
\hline 03. Facilidade de uso & & 0,09 & 0,08 & 1,09 & H7 - Rejeitada \\
\hline 04. Adequação à tecnologia & & 0,69 & 0,11 & 6,50 & H8 - Suportada \\
\hline 01. Características da Tarefa & \multirow{4}{*}{$\begin{array}{l}07 . \\
\text { Comprometimento }\end{array}$} & 0,02 & 0,11 & 0,16 & H9 - Rejeitada \\
\hline 02. Características da Tecnologia & & 0,35 & 0,11 & 3,18 & H10 - Suportada \\
\hline 03. Facilidade de uso & & 0,13 & 0,12 & 1,07 & H11 - Rejeitada \\
\hline 04. Adequação à tecnologia & & 0,31 & 0,12 & 2,53 & H12 - Suportada \\
\hline 01. Características da Tarefa & \multirow{5}{*}{ 08. Confiança } & $-0,12$ & 0,11 & 1,05 & H13 - Rejeitada \\
\hline 02. Características da Tecnologia & & 0,10 & 0,17 & 0,58 & H14 - Rejeitada \\
\hline 03. Facilidade de uso & & 0,10 & 0,13 & 0,75 & H15 - Rejeitada \\
\hline 04. Adequação à tecnologia & & 0,40 & 0,14 & 2,81 & H16 - Suportada \\
\hline 05. Utilidade & & 0,22 & 0,14 & 1,57 & H17 - Rejeitada \\
\hline 05. Utilidade & 09. Propensão & $-0,21$ & 0,11 & 1,86 & H18 - Suportada \\
\hline (C) RDBCI: Rev. Digit. Bibliotecon. Cienc. Inf. & Campinas, SP & v. 15 & n.1 & $189-210$ & jan./abr. 2017 \\
\hline
\end{tabular}




\begin{tabular}{llllll} 
06. Satisfação & lealdade & 0,12 & 0,12 & 1,00 & H19 - Rejeitada \\
07. Comprometimento & & 0,56 & 0,11 & $\mathbf{5 , 2 1}$ & H20 - Suportada \\
08. Confiança & & 0,24 & 0,13 & 1,94 & H21 - Rejeitada \\
\hline 05. Utilidade & & 0,04 & 0,12 & 0,36 & H22 - Rejeitada \\
06. Satisfação & \multirow{2}{*}{ Comunicação } & $-0,05$ & 0,09 & 0,57 & H23 - Rejeitada \\
07. Comprometimento & positiva & 0,60 & 0,13 & $\mathbf{4 , 5 3}$ & H24 - Suportada \\
08. Confiança & & 0,22 & 0,12 & 1,84 & H25 - Rejeitada \\
\hline \hline
\end{tabular}

Fonte: Dados da pesquisa.

Observações: a) Peso: é o peso padronizado obtido para amostra completa; b) Erro: é o erro estimado da estimativa; c) Valor T: é a razão entre o peso não padronizado pelo seu erro padrão.

O construto " 05 . Utilidade" apresentou um $\mathrm{R}^{2}$ de $61 \%$, sendo que os construtos " 01 . Características da Tarefa" (carga de 0,03 e p valor $>10 \%$ ) e "03. Facilidade de uso" (carga de 0,04 e p valor $>10 \%$ ) não apresentaram impacto estatisticamente significativo no mesmo. Já os construtos "02. Características da Tecnologia" (carga de 0,54 e p valor $<1 \%$ ) e "04. Adequação à tecnologia" (carga de 0,34 e p valor <1\%) apresentaram impactos estatisticamente significativos e positivos, sendo que o primeiro apresentou uma carga de maior magnitude.

O construto "06. Satisfação" apresentou um $\mathrm{R}^{2}$ de $63 \%$, sendo que os construtos " 01 . Características da Tarefa" (carga de 0,04 e p valor $>10 \%$ ) e "03. Facilidade de uso" (carga de 0,09 e p valor $>10 \%$ ) também não apresentaram impacto estatisticamente significativo. Paralelamente, os construtos "02. Características da Tecnologia" (carga de 0,20 e p valor $<5 \%$ ) e "04. Adequação à tecnologia" (carga de 0,69 e p valor $<1 \%$ ) apresentaram impactos estatisticamente significativos e positivos, sendo o segundo de maior magnitude.

O construto "07. Comprometimento" apresentou um $\mathrm{R}^{2}$ um pouco inferior, de $31 \%$, sendo que, nesse caso, os construtos "01. Características da Tarefa" (carga de 0,02 e $\mathrm{p}$ valor $>10 \%$ ) e "03. Facilidade de uso" (carga de 0,13 e p valor $>10 \%$ ) também não apresentaram impacto estatisticamente significativo. Em contrapartida, os construtos " 02 . Características da Tecnologia" (carga de 0,35 e p valor $<1 \%$ ) e "04. Adequação à tecnologia" (carga de $0,31 \mathrm{e} \mathrm{p}$ valor $<1 \%$ ) apresentaram impactos estatisticamente significativos e positivos, sendo a magnitude de ambos bem similar.

No caso do construto "08. Confiança", o $\mathrm{R}^{2}$ apresentado foi de $43 \%$, sendo que os construtos que apresentaram impacto estatisticamente significativo e positivo foram o " 04 . Adequação à tecnologia" (carga de 0,40 e $p$ valor $<1 \%$ ) e o " 05 . Utilidade" (carga de 0,22 e $p$ valor $<10 \%$ ). Já os construtos " 01 . Características da Tarefa" (carga de $-0,12$ e p valor $>10 \%$ ), "02. Características da Tecnologia" (carga de 0,10 e p valor $>10 \%$ ) e "03. Facilidade de uso" (carga de 0,10 e p valor $>10 \%$ ) não apresentaram impactos estatisticamente significativos.

No caso do construto " 09 . Propensão a manter o relacionamento", o $\mathrm{R}^{2}$ foi de $54 \%$, sendo que o único construto que não apresentou impacto estatisticamente significativo foi o "06. Satisfação" (carga de 0,12 e p valor $>10 \%$ ). Os outros três "05. Utilidade" (carga de -

\begin{tabular}{|c|}
\hline RDBCI: Rev. Digit. Bibliotecon. Cienc. Inf. \\
\hline
\end{tabular}


0,21 e p valor $<10 \%$ ), "07. Comprometimento" (carga de 0,56 e p valor $<1 \%$ ) e "08. Confiança" (carga de 0,24 e p valor $<5 \%$ ) apresentaram impactos estatisticamente significativos. O Comprometimento e a Confiança apresentaram impactos positivos, enquanto a Utilidade apresentou um impacto negativo, revelando que quanto maior a utilidade, menor a lealdade, o que vai contra o que foi hipotetizado. Vale ressaltar que o seu T crítico foi significativo ao nível de $10 \%$, existindo uma possibilidade de tal sinal ter decorrido de erros amostrais. Por fim, o construto que apresentou a maior magnitude foi o "07. Comprometimento", indicando ser o que possui maior relação com a lealdade. Os outros dois apresentaram cargas de magnitudes bem semelhantes.

Por fim, o construto " 10 . Comunicação positiva" apresentou um $\mathrm{R}^{2}$ de $59 \%$, sendo que a "05. Utilidade" (carga de 0,04 e p valor >10\%) e a "06. Satisfação" (carga de -0,05 e p valor $>10 \%$ ) não apresentaram impacto estatisticamente significativos. Já o "07. Comprometimento" (carga de 0,60 e p valor $<1 \%$ ) e a "08. Confiança" (carga de 0,22 e p valor $<5 \%$ ) se mostraram estatisticamente significantes e positivos, sendo que o primeiro apresentou uma carga de maior magnitude.

É importante verificar também a qualidade do ajuste. Para tanto, pode ser utilizado o Goodness of fit $(\mathrm{GoF})$, que pode ser calculado com base na fórmula proposta por Amato, Vinzi e Tenenhaus (2004), que é operacionalizada da seguinte maneira: devem ser verificadas as médias das AVEs e dos $\mathrm{R}^{2}$ dos construtos do modelo e, em sequência, verificar a média geométrica, que dará o GoF do modelo. Tal valor varia de $0 \%$ a $100 \%$, não havendo ainda valores de corte para considerar um ajuste como bom ou ruim. Entretanto, quanto mais próximo de $100 \%$ melhor o ajuste, sendo que o GoF do modelo foi de $60 \%$ o que indica que a média de variabilidade prevista no modelo foi de 60\%. Apesar de não existir critérios absolutos de qualidade para esta medida (VINZI; TRINCHERA; AMATO, 2010) e seu uso ser controverso (HAIR et al., 2014), seu valor supera o limite de 50\% tipicamente proposto (HULLAND, 1999).

Além disso, deve ser avaliada, no Inner Path Model, a capacidade de predição do modelo por meio da medida Stone-Geisser's $Q^{2}$, que postula que o modelo deve ser capaz de predizer adequadamente os construtos endógenos conforme sugerem Hair, Ringle e Sarstedt (2011). No SmartPLS, essa medida é obtida por meio do procedimento denominado Blindfolding, sendo que o valor $d$ requisitado pelo teste deve estar entre 5 e 10 , conforme sugerem os autores, e atestam que a medida denominada cross-validated redundancy deve ser preferida à medida denominada crossvalidated communality. Henseler, Ringle e Sinkovics (2009) revelam que, quando a medida é superior a 0\%, o construto endógeno possui um poder de previsão relevante. Para tanto, foi adotado um $d$ de sete e realizada tal análise, sendo que a TAB. 19 exibe o resultado para os construtos do modelo. Os resultados revelam que todos os construtos endógenos do modelo apresentam um $Q^{2}$ superior a $0 \%$, o que indica que os construtos endógenos foram adequadamente mensurados pelo modelo. 
Tabela 3. Ajuste geral das medidas

\begin{tabular}{l|l}
\hline \hline Construtos endógenos & $\boldsymbol{Q}^{\mathbf{2}}$ \\
\hline 05. Utilidade & $36 \%$ \\
06. Satisfação & $45 \%$ \\
07. Comprometimento & $22 \%$ \\
08. Confiança & $33 \%$ \\
10. Propensão a manter o relacionamento & $38 \%$ \\
11. Comunicação positiva & $51 \%$ \\
Média dos construtos do modelo & $\mathbf{3 8 \%}$ \\
\hline \hline
\end{tabular}

Fonte: Dados da pesquisa.

\section{CONSIDERAÇõES FINAIS}

Esta pesquisa teve como objetivo avaliar o papel da interação da tecnologia como fomento do relacionamento, a partir da conjugação de modelos relacionais (KMV) e de Adoção da Tecnologia (TAM e TTF). Ao analisar os resultados das hipóteses, pode-se dizer que o construto Característica da Tarefa não se apresentou como um antecedente da satisfação, do comprometimento, da utilidade e da confiança, isso é, a presença (ou ausência) de tarefas complexas e interdependentes para solução de problemas acadêmicos e administrativos na instituição pesquisada não exerceu impacto sobre as variáveis relacionais chave do modelo. Cabe ressaltar que as médias dos indicadores destes construtos é inferior a 3 , indicando que problemas desta natureza são percebidos como raros.

Já a característica da tecnologia afeta a satisfação, o comprometimento e a utilidade percebida pelo usuário do portal, de modo que uma avaliação positiva da tecnologia, em elementos como interface, conteúdo, tamanho do site, serviços que dispõe para o usuário e ferramentas digitais, são fundamentais para aproximar o aluno da instituição, muito além da mera utilização do sistema. Cabe destacar que a "característica da tecnologia" não explicou estatisticamente o constructo confiança.

A facilidade de uso não explica utilidade, confiança, satisfação e comprometimento, bem como não impactou na utilização de um site e sistema. Suspeita-se pelas elevadas médias deste construto que os usuários, de perfil jovem, não sintam dificuldades e sejam versados na utilização de tecnologias similares dentro e fora do âmbito acadêmico, determinando uma maior homogeneidade e menor impacto da facilidade de uso como fomento ao relacionamento entre a instituição e seu público.

O construto "adequação à tecnologia" do modelo TTF apresentou um impacto positivo em todas as suas relações. Neste ponto, a avaliação da adequação do portal às necessidades especificadas de cada aluno no cotidiano acadêmico explica em grande parte não só o uso do sistema como as bases do relacionamento (satisfação, comprometimento e confiança). 
A propensão em manter um relacionamento foi de 54\%, explicada positivamente pelo comprometimento e pela confiança, enquanto a satisfação não foi significativa, conforme esperado pelo modelo KMV, além de sofrer efeito direto da utilidade. A comunicação positiva foi explicada em 59\% pela confiança e pelo comprometimento (H25).

De forma geral, observa-se que a tecnologia apresenta papel fundamental para fomentar os relacionamentos na instituição pesquisada, revelando a importância de se ter um portal compatível às atividades e relações de longo prazo com os clientes alunos. De forma mais genérica, propõe-se que elementos tecnológicos, em especial aqueles associados a era digital, firmam-se progressivamente como parte integrante e indissociável no fortalecimento dos laços entre clientes e organizações, sob a ótica do marketing de relacionamento. Portanto, deve-se atentar para o planejamento de um site e de seus sistemas a partir da expectativa do usuário, ressaltando a importância de se conhecer o perfil e rotina de atividades para que o investimento em tecnologia digital se torne uma ferramenta de relacionamento efetiva.

Em especial, no caso das IES que lidam com um público que rapidamente se adapta a questões tecnológicas e pode rapidamente aprender como um portal pode ser utilizado para a gestão de inúmeras atividades no cotidiano, a preocupação central dos desenvolvedores é encontrar pontos de interseção em funcionalidades e aplicativos que visem aproximar o aluno da IES. Nitidamente, questões como usabilidade e facilidade de uso parecem ser secundárias a este público, com impactos não significativos ao fomento de relacionamentos. Já o projeto do sistema e sua aplicação pela comunidade como ferramenta de aproximação da tríade aluno-docente-instituição e de criação de valor compartilhado, possivelmente transcendendo questões administrativas e da gestão de sala de aula, merecem destaque especial.

Dentre as limitações destes achados, destacam-se a amostra não probabilística, que limita generalizações e a realização apenas em uma organização do segmento educacional. A análise de dados aplicada pela abordagem PLS, também não permite averiguar a aderência do modelo como um todo, somente a capacidade preditiva. Assim, para testar o modelo com maior robustez, seria necessário buscar uma maior amostra e, talvez, o emprego de métodos robustos a desvios de estimação utilizando a análise estrutural pela abordagem de covariância, utilizando os índices típicos de aderência do modelo.

Sugere-se estender as relações aqui propostas para outros cenários, variando características como idade (neste caso caracterizado por faixas etárias jovens), renda (neste estudo de renda elevada), grau de envolvimento (trata-se de um setor em que os vínculos são relativamente estáveis) e afinidade com a tecnologia. 


\section{MARKETING DIGITAL Y RELACIÓN MARKETING: EVALUACIÓN DE UN SISTEMA DE INFORMACIÓN DIGITAL MEDIANTE UNA INTEGRACIÓN DE MODELOS KMV TAM, E TTF}

RESUMEN: La creciente popularidad de Internet prolifera formas de entretenimiento, la comunicación y el trabajo, tales como portales, cuyo objetivo es difundir la información y las relaciones de crianza entre las partes interesadas. Este estudio tuvo como objetivo verificar como portal de interacción tecnológica del IES afecta a las actitudes (satisfacción, confianza y compromiso) y las intenciones de comportamiento (uso del portal, la lealtad y la comunicación positiva) de los estudiantes. A partir de una encuesta diseñada por modelos TAM, KMV TTF y el estudio muestra Que las características y la idoneidad de la tecnología de la influencia de la utilidad percibida en el portal y en la moderación de las actitudes de las intenciones de comportamiento de la institución estudiada.

PALABRAS CLAVE: Portal; Digital, Evaluación del sistema de recuperación de información

Submetido em: 09-06-2016

Aceito em: 15-12-2016

Publicado em: 15/12/2016

\section{REFERÊNCIAS}

AGUSTINI, Anapatricia M. Vilha di; MINCIOTTI, Silvio Augusto. Envolvimento do Consumidor: Análise e Aplicações dos Conceitos em Situação de Compra pela internet. FEA/USP: São Paulo, 2003.

ALEJANDRO, Thomas Brashear et al. The outcome of company and account manager relationship quality on loyalty, relationship value and performance. Industrial Marketing Management, v. 40, n. 1, p. 36-43, 2011. Disponível em:

<http://linkinghub.elsevier.com/retrieve/pii/S0019850110001677>. Acesso em: 7 mar. 2013.

ALVES, Mauro Halfeld Ferrari; LAMOUNIER, Ana Elisa Bacha; JABUR. Fábio Pinheiro. Internet - adicionando valor por meio de experiências descontínuas: a experiência brasileira. RAUSP, 1999. Disponível em: <http://www.rausp.usp.br/>. Acesso em 3 out. 2011.

DO AMARAL, Sueli Angelica. Gestão da informação e do conhecimento nas organizações e orientação de marketing. Informação \& Informação, v. 13, n. 1esp, p. 52-70, 2008.

TENENHAUS, Michel; AMATO, Silvano; ESPOSITO VINZI, V. A global goodness-of-fit index for PLS structural equation modelling. In: Proceedings of the XLII SIS scientific meeting. 2004. p. 739-742.

ANDERSON, James C.; GERBING, David W. Structural equation modeling in practice: A review and recommended two-step approach. Psychological bulletin, v. 103, n. 3, p. 411, 
1988. Disponível em: <http://doi.apa.org/getdoi.cfm?doi=10.1037/0033-2909.103.3.411>. Acesso em: 29 mar. 2012.

BALARINE, Oscar Fernando Osorio. Tecnologia da informação como vantagem competitiva. RAE-eletrônica, v. 1, n. 1, jan./jun. 2002.

CARVALHO, Antonino Felicio dos Santos de; CARVALHO, Frederico Antonio Azevedo de; CARVALHO, José Luis Felício. Estudo empírico sobre conteúdo promocional em websites de grandes empresas brasileiras: revisitando Soares \& Hoppen. Rio de Janeiro, 2007.

CHIN, Wynne W. Frequently asked questions-partial least squares \& PLS-graph., v. 15, p. 2006, 2000. Disponível em: <http://disc-nt.cba.uh.edu/chin/plsfaq.htm>. Acesso em: 6 jun. 2012.

DAVIS, Fred D. User acceptance of information technology: system characteristics, user perceptions and behavioral impacts. International journal of man-machine studies, v. 38, n. 3, p. 475-487, 1993. International Journal of Man-Machine Studies, v. 38, n. 3, p. 475-487, mar. 1993.

DAVIS, Fred. Perceived usefulness, perceived ease of use and user acceptance of information technology. MIS Quarterly, v.13, n.3, p.319-339, set. 1989.

DISHAW, Mark T.; STRONG, Diane M. Extending the technology acceptance model with task-technology fit constructs. Information \& management, v. 36, n. 1, p. 9-21, 1999.

DWYER, F. Robert; SCHURR, Paul H.; OH, Sejo. Developing buyer-seller relationships. The Journal of marketing, p. 11-27, 1987.

FARIAS, Salomão Alencar de. Atmosfera de loja on-line: o impacto do ambiente virtual na satisfação do consumidor e na atitude para com a compra. RAUSP, 2006. Disponível em: <http://www.rausp.usp.br/>. Acesso em 27 jul. 2010.

AJZEN, Icek; FISHBEIN, Martin. Belief, attitude, intention and behavior: An introduction to theory and research. 1975.

FORNELL, Claes; LARCKER, David F. Evaluating Structural Equation Models with Unobservable Variables and Measurement Error. Journal of Marketing Research, v. 18, n. 1, p. 39, fev. 1981. Disponível em: <http://www.jstor.org/stable/10.2307/3151312〉. Acesso em: 14 jun. 2012.

GOODHUE, Dale L.; THOMPSON, Ronald L. Task-technology fit and individual performance. MIS quarterly, p. 213-236, 1995.

GUMMESSON, E. Total relationship marketing: rethinking marketing management. Second ed.Oxford: Elsevier Science, 1999.

GUMMESSON, Evert. Total relationship marketing. 2nd.ed. Oxford Amsterdam Boston London New York Paris San Diego San Francisco Singapore Sydney Tokyo. Oxford: Butterworth-Heinemann, 2002. 
HAIR, J. F. J. et al. A Primer on Partial Least Squares Structural Equation Modeling (PLSSEM). London, SAGE, 2014.

HAIR, J. F.; RINGLE, C. M.; SARSTEDT, M. PLS-SEM: Indeed a Silver Bullet. Journal of Marketing Theory and Practice, v.19, n.2, p.139-151, 2011.

HAIR, Joseph F. et al. Multivariate Data Analysis. 7. ed. New York, USA: Prentice Hall, 2010. p. 816

HENSELER, Jorg; RINGLE, Christian M.; SINKOVICS, Rudolf R. The Use Of Partial Least Squares Path Modeling In International Marketing. Advances in International Marketing, v. 20, p. 277-319, 2009.

HERNANDEZ, José Mauro da Costa. Desenvolvimento e validação de medidas da confiança em comércio eletrônico: uma nova tipologia multidisciplinar, 2008. III ENCONTRO ANPAD, 2008. Curitiba, Anais..., Curitiba, 2008.

HOLLENSEN, S. Marketing Management: A Relationship Approach. 2. ed. Harlow: FT Prentice Hall, 2010.

HULLAND, John. Use of partial least squares (PLS) in strategic management research: a review of four recent studies. Strategic Management Journal, v. 20, n. 2, p. 195-204, fev. 1999.

PRIES, Kim H.; DUNNIGAN, Robert. Big Data Analytics: A practical guide for managers. CRC Press, 2015.

KLOPPING, Inge M.; MCKINNEY, Earl. Extending the technology acceptance model and the task-technology fit model to consumer e-commerce. Information Technology, Learning, and Performance Journal, v. 22, n. 1, p. 35, 2004.

LACERDA, Tales Sarmento,; MENDONÇA, Beatriz Quaresma. Usabilidade e atendimento ao usuário na web: uma análise heurística dos sites de associações e publicações acadêmicas de administração no Brasil. In: II ENCONTRO DE ADMINISTRAÇÃO DA INFORMAÇÃ̃O, 2009.

LEEFLANG, Peter SH et al. Challenges and solutions for marketing in a digital era. European management journal, v. 32, n. 1, p. 1-12, 2014.

MARTINEZ, Maria Laura; FERREIRA, Sueli Mara S. P.; GALINDO, Marcos. Estudo de usabilidade do Portal de Periódicos da Capes: análise de perfil do usuário discente da UFPE. 2011. Disponível em:

http://www2.capes.gov.br/rbpg/images/stories/downloads/RBPG/Vol.8_15/3_Artigo.pdf. Acesso em 15 de janeiro de 2012.

MORAES, Marcelo Amaral de. Comércio eletrônico: uma análise da qualidade, satisfação e intenções comportamentais dos consumidores de supermercados virtuais de Minas Gerais. 2002. Dissertação (Mestrado) - Faculdade de Ciências Econômicas da UFMG, Belo Horizonte, 2002. 
MORGAN, Robert M.; HUNT, Shelby D. The commitment-trust theory of relationship marketing. The journal of marketing, p. 20-38, 1994. Disponível em:

$\langle$ http://www.jstor.org/stable/1252308?origin=crossref $>$.

NAKAGAWA, Sandra Sayuri Yamashita; GOUVÊA Maria Aparecida. Marketing de relacionamento sobre a influência da internet. Revista de Gestão USP, São Paulo, v. 13, n. 1, p. 57-73, janeiro/março 2006. Disponível em:

<www.revistasusp.sibi.usp.br/pdf/rege/v13n1/v13n1a5.pdf>. Acesso em 30 set. 2011.

NETEMEYER, Richard G.; BEARDEN, William O.; SHARMA, Subhash. Scaling procedures: Issues and applications. Sage Publications, 2003.

NIELSEN, Jakob; LORANGER, Hoa. Usabilidade na web: projetando websites com qualidade. Tradução de Edson Furmankiewicz e Carlos Schafranski. Rio de Janeiro: Elsevier, 2007.

OKADA, Sionara Ioco; DE SOUZA, Eliane Moreira Sá. Estratégias de marketing digital na era da busca. REMark, v. 10, n. 1, p. 46, 2011.

PARVATIYAR, Atul; SHELTH, Jagdish N. Customer relationship management: emerging practice, process, and discipline. Journal of Economic and Social Research, v.3, n.2, p.1-34, 2001.

SALEH, Amir Mostafa. Adoção de Tecnologia: Um estudo sobre o uso de software livre nas empresas. 2004. Dissertação (Mestrado) - Faculdade de Economia, Administração e Contabilidade da USP, São Paulo, 2004.

TERRA, José Cláudio C. Gestão 2.0: como integrar a colaboração e a participação em massa para o sucesso do negócio. Rio de Janeiro: Elsevier, 2010.

TERRA, José Cláudio Cyrineu; GORDON, Cindy. Portais corporativos: a revolução na gestão do conhecimento. 3.ed. São Paulo: Negócio Editora Ltda, 2002.

DA SILVA TOLENTINO, Renata de Sousa et al. Avaliação da Qualidade na Educação a Distância sob a perspectiva do Aluno: Proposição e teste de um modelo usando equações estruturais. REGE Revista de Gestão, v. 20, n. 3, p. 347-366, 2013.

TOLENTINO, Renata Sousa et al. ANÁLISE DO DESEMPENHO INDIVIDUAL DE USUÁRIOS DE SISTEMAS DE INFORMAÇÃO: UM ESTUDO INTEGRATIVO DOS MODELOS TAM E TTF. Revista de Administração FACES Journal, v. 6, n. 2, 2007.

TORRES, Cláudio. A bíblia do marketing digital. Tudo o que você queria saber do marketing e não tinha a quem perguntar. São Paulo: Novatec, 2009.

VIEIRA, Valter Afonso; MONTEIRO, Plinio R.R.; VEIGA, Ricardo Teixeira. Relationship marketing in supply chain: an empirical analysis in the Brazilian service sector. Journal of Business \& Industrial Marketing, v. 26, n. 7, p. 524-531, 2011. Disponível em: <http://www.emeraldinsight.com/10.1108/08858621111162325>. Acesso em: 14 nov. 2013.

VINZI, Vincenzo Esposito; TRINCHERA, Laura; AMATO, Silvano. PLS path modeling: from foundations to recent developments and open issues for model assessment and 
improvement. In: Handbook of partial least squares. Springer Berlin Heidelberg, 2010. p. 47-82.

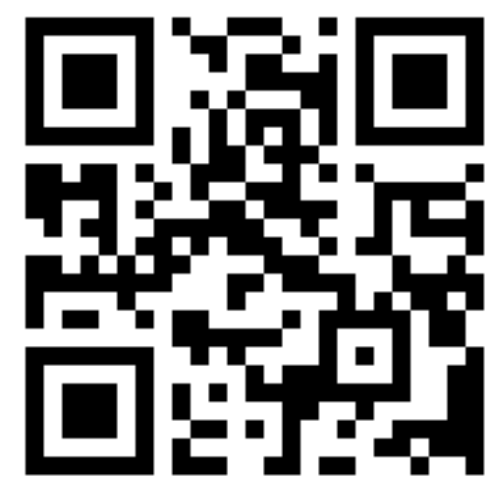

